\title{
Heat shock protein peptide complex-96 vaccination for newly diagnosed glioblastoma: a phase I, single-arm trial
}

\author{
Nan Ji, Yang Zhang, Yunpeng Liu, Jian Xie, Yi Wang, Shuyu Hao, and Zhixian Gao \\ Department of Neurosurgery, Beijing Tiantan Hospital, Capital Medical University, Beijing, China. China National Clinical \\ Research Center for Neurological Diseases, Beijing, China.
}

BACKGROUND. Heat shock protein peptide complex-96 (HSPPC-96) triggers adaptive and innate antitumor immune responses. The safety and efficacy of HSPPC-96 vaccination was examined in patients with newly diagnosed glioblastoma multiforme (CBM).

METHODS. In this open-label, single-arm, phase I study, adult patients were vaccinated with HSPPC-96 in combination with the standard treatment for newly diagnosed CBM after surgical resection. Primary endpoints were frequency of adverse events and progression-free survival (PFS) at 6 months. Secondary endpoints included overall survival (OS), PFS, and tumor-specific immune response (TSIR).

RESULTS. A total of $\mathbf{2 0}$ patients with newly diagnosed CBM were enrolled from September 2013 to February 2015. No grade 3 or 4 vaccine-related adverse events were noted. After a median follow-up of 42.3 months, PFS was $89.5 \%$ ( $95 \% \mathrm{Cl}, 66.9 \%-98.7 \%)$ at 6 months, median PFS was 11.0 months (95\% Cl, 8.2-13.8), and median OS was 31.4 months (95\% Cl, 14.9-47.9). TSIR was significantly increased by 2.3 -fold ( $95 \% \mathrm{Cl}, 1.7-3.2$ ) after vaccination. Median OS for patients with high TSIR after vaccination was $>\mathbf{4 0 . 5}$ months $(95 \% \mathrm{Cl}$, incalculable) as compared with 14.6 months $(95 \%$ $\mathrm{CI}, 7.0-22.2$ ) for patients with low TSIR after vaccination (hazard ratio, $0.25 ; 95 \% \mathrm{Cl}, 0.071-0.90$; $P=0.034)$. A multivariate Cox regression model revealed TSIR after vaccination as a primary independent predicator for survival.

CONCLUSION. The HSPPC-96 vaccination, combined with the standard therapy, is a safe and effective strategy for treatment of newly diagnosed CBM patients. TSIR after vaccination would be a good indicator predicting the vaccine efficacy.

TRIAL REGISTRATION. ClinicalTrials.gov NCT02122822.

FUNDING. National Key Technology Research and Development Program of the Ministry of Science and Technology of China (2014BAI04B01, 2014BAI04B02), Beijing Natural Science Foundation (7164253), Beijing Talents Fund (2014000021469G257), and Shenzhen Science and Technology Innovation Committee (JSGG20170413151359491).

Authorship note: NJ and YZ contributed equally to this work.

Conflict of interest: The authors have declared that no conflict of interest exists.

Submitted: December 13, 2017

Accepted: April 12, 2018

Published: May 17, 2018

Reference information: JCI Insight. 2018;3(10):e99145. https://doi.org/10.1172/jci. insight. 99145

\section{Introduction}

Glioblastoma multiforme (GBM), the most common and invasive primary brain malignancy, is universally associated with a dismal prognosis $(1,2)$. The medial survival time is no more than 15 months, and 5-year survival rate is less than 10\% in newly diagnosed GBM patients, who receive standard treatment, including maximal safe surgical resection, radiotherapy, and systemic chemotherapy $(1,2)$. Therefore, new therapeutic modalities are urgently needed to improve the poor outcomes of GBM patients.

Immunotherapy has exhibited impressive antitumor activity in several cancers $(3,4)$ and would offer opportunities for better outcomes in GBM patients (5-7). HSPs are a family of proteins, named for their role in cellular responses to stressors such as heat. HSPs function as intracellular chaperons and bind tumor-associated antigens (peptides). HSP-peptide complexes can be taken up by antigen-presenting cells and then trigger specific antitumor responses $(8,9)$. HSPs also act as adjunctives by boosting innate immune response during the tumor-antigen-presenting process $(8,10)$. Therefore, after a simple purification of HSP-peptide 


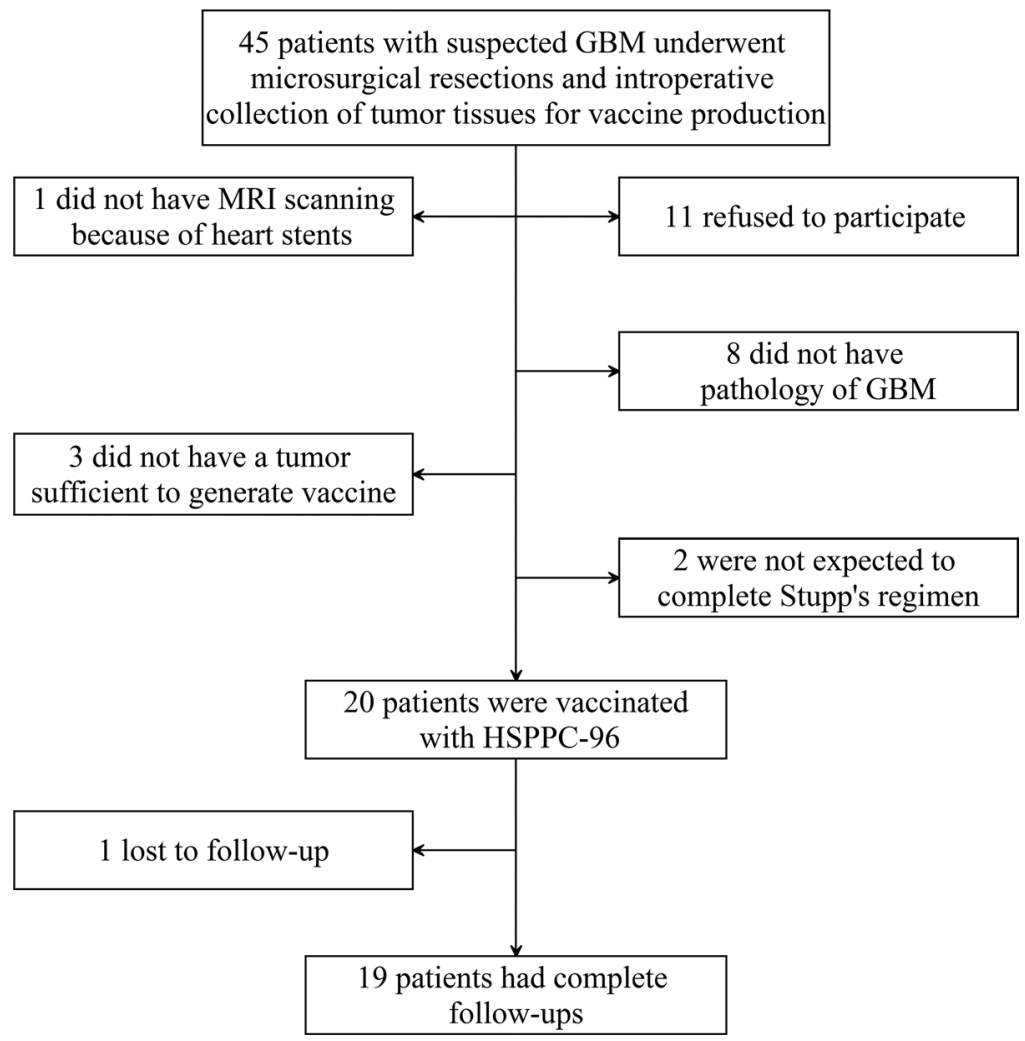

Figure 1. Overview of patient flow and disposition in this trial. complexes from a patient's tumor, the complexes can be directly administered as a personalized polyvalent antitumor vaccine (11). Among vaccines of this type, heat shock protein peptide complex-96 (HSPPC-96) is the most widely used to treat gliomas (11).

The safety and efficacy of HSPPC-96 vaccination has been demonstrated in a group of patients with recurrent GBM $(12,13)$. The initial phase I trial indicated that HSPPC-96 vaccination can trigger specific immune responses at peripheral and tumor sites, without adverse events related to the vaccination (12). The subsequent phase II trial enrolled 41 patients who had undergone gross total resection of the recurrent tumor (13). After administration of a median of 6 doses of the HSPPC-96 vaccine, survival was $90.2 \%$ at 6 months and $29.3 \%$ at 12 months. No treatment-related deaths were observed, and only a single grade 3 constitutional event related to the vaccine was reported (13). Based on these promising results in patients with recurrent GBM, we herein aim to evaluate the safety and preliminary efficacy of the HSPPC-96 vaccine in patients with newly diagnosed GBM.

\section{Results}

Basic characteristics of the included patients. A total of 20 patients with newly diagnosed supratentorial GBM were included in the study (Figure 1). All patients underwent microsurgery at the Beijing Tiantan Hospital from September 2013 to February 2015 and received the standard

Stupp regimen of radiotherapy plus concomitant and adjuvant temozolomide (TMZ) (1). Gross total resection was achieved in all the patients (Table 1). During adjuvant TMZ chemotherapy, 6 doses of the HSPPC-96 vaccine were administered to each patient (Figure 2, detailed in the Methods). The median time from surgery to the first dose of vaccine was 98 days (range, 88-128 days). The median follow-up time was 42.3 months (range, 34.6-51.6 months). At the time of the last follow-up, 16 patients underwent tumor progression and 11 were dead. Two molecular biomarkers of significant importance for GBM involve isocitrate dehydrogenase (IDH) mutations and O6-methylguanine-DNA methyltransferase (MGMT) promoter methylation. Both are associated with improved prognosis for GBM patients (13-16). For better evaluation of outcomes in this GBM cohort, IDH mutations and MGMT promoter methylations (meMGMTs) were surveyed in the 16 cases with residual tumor samples. Of these, only 2 cases were found to have meMGMT or IDH mutations (Table 1).

Safety. Several slight adverse events were observed in patients after one or more doses of the HSPPC-96 vaccine (Table 2). Regarding vaccine-related adverse events, fatigue was the most common adverse event (4, 20\%). One patient had low fever after the third HSPPC-96 administration, and another patient had cutaneous pruritus after the second administration. All the symptoms resolved spontaneously within 72 hours after vaccine administration. Only one patient developed a grade 3 adverse event (focal neurological deficit: hemiplegia) during the treatment. The exact source for the hemiplegia was unclear, but it might have been caused by a delayed infarction incurred during radiotherapy or surgery.

Clinical activity and immunological response. Across the 19 patients with complete follow-ups (Figure 1), progression-free survival (PFS) was $89.5 \%$ (95\% CI, 66.9\%-98.7\%) at 6 months, median PFS was 11.0 months (95\% CI, 8.2-13.8), and median overall survival (OS) was 31.4 months (95\% CI, 14.9-47.9) (Figure 3). Tumor-specific immune response (TSIR) was evaluated by the number of stimulated peripheral blood mononuclear cells (PBMCs) in response to autologous tumor lysate in an IFN- $\gamma$ release enzymelinked immunospot (ELISPOT) assay. The average number was 29.86 (95\% CI, 17.20-51.86) spots/3 $\times$ $10^{5}$ PBMCs before vaccination and 68.66 (95\% CI, 42.26-111.54) spots $/ 3 \times 10^{5} \mathrm{PBMCs}$ after vaccination (Figure 4). TSIR was significantly increased by 2.3 -fold $(95 \% \mathrm{CI}, 1.7-3.2, P<0.0001)$ after vaccination. 
Table 1. Basic characteristics of all treated patients

\begin{tabular}{|c|c|c|}
\hline & No. & Proportion (\%) \\
\hline \multicolumn{3}{|l|}{ Sex } \\
\hline Male & 10 & 50 \\
\hline Median age at diagnosis, yr (range) & $52(40-70)$ & \\
\hline Median preoperative KPS (range) & $80(70-100)$ & \\
\hline \multicolumn{3}{|l|}{ Surgical status } \\
\hline \multicolumn{3}{|l|}{ Salvage therapy after progression ${ }^{A}$} \\
\hline Radiosurgery & 6 & 37.5 \\
\hline Bevacizumab & 5 & 31.3 \\
\hline Reresection & 2 & 12.5 \\
\hline No tumor-specific therapy & 6 & 37.5 \\
\hline \multicolumn{3}{|l|}{ IDH $1 / 2$ mutations } \\
\hline Mutated & 2 & 10 \\
\hline Wild-type & 14 & 70 \\
\hline Unknown & 4 & 20 \\
\hline \multicolumn{3}{|l|}{ Status at last follow-up: } \\
\hline Alive & 8 & 40.0 \\
\hline Dead & 11 & 55.0 \\
\hline Unknown & 1 & 5.0 \\
\hline Median follow-up time, mo (range) & $42.3(34.6-51.6)$ & \\
\hline
\end{tabular}

This indicated that the antitumor immune response was stimulated by the HSPPC-96 vaccination.

Furthermore, TSIR varied in each patient during the course of the vaccinations (Figure 5A). Based on the TSIR after vaccination, we divided the included patients into a high TSIR group (TSIR $\geq$ median) and a low TSIR group (TSIR < median) (Figure 5A). Baseline characteristics were quite similar between the two groups (Supplemental Table 1; supplemental material available online with this article; https://doi.org/10.1172/jci.insight.99145DS1). Median OS for patients with high TSIR was $>40.5$ months (95\% CI, incalculable) as compared with 14.6 months (95\% CI, 7.0-22.2) for patients with low TSIR (hazard ratio, 0.25; 95\% CI, 0.071-0.90; $P=0.034$ ) (Figure 5C). Median PFS in the high TSIR group was 12.3 months (95\% CI, 7.7-16.9), which is longer than the 9.0-month PFS (95\% CI, 7.6-10.4) for patients with low TSIR (hazard ratio, $0.32 ; 95 \% \mathrm{CI}, 0.11-0.94 ; P=0.038$ ) (Figure $5 \mathrm{~B}$ ). After vaccination TSIR also emerged as a significant independent prognostic indicator from a Cox regression model $(P=0.011$; Table 3$)$. The adjusted hazard ratio for TSIR after vaccination was 0.11 in our patients (95\% CI, 0.02-0.60; Table 3). In contrast, the baseline state of TSIR (TSIR before vaccination) did not effect the outcomes for patients (Supplemental Figure 1).

\section{Discussion}

GBM is notorious for tumor-associated immunosuppression $(5,7,11)$, involving multiply cellular interactions and molecular pathways $(17,18)$. Accordingly, numerous strategies have been explored to overcome this immunosuppression and to promote antitumor immune response. As one of these strategies, vaccination approaches have been extensively investigated in rodent models and in large clinical trials for the treatment of glioma $(11,19)$. HSPPC-96, a tumor-derived peptide vaccine, has been identified as a safe and potentially effective immunotherapy for the treatment of several late-stage human malignancies, including melanoma $(20,21)$, renal cell carcinoma $(20,22)$, colorectal cancer 


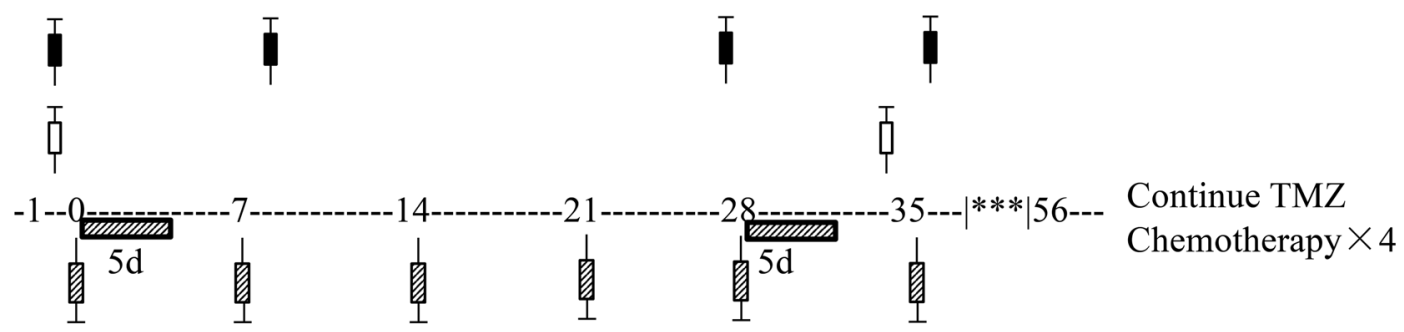

Blood collected for routine/biochemical test $\coprod_{T}$ Blood collected for IFN- $\gamma$ release ELISPOT שm TMZ adjuvant chemotherapy (150-200 mg/m²/day, p.o.)

首 Vaccine $(25 \mu \mathrm{g}$, s.c. $)+\operatorname{CTX}(400 \mathrm{mg}, \mathrm{i} . \mathrm{v}$.

Figure 2. Scheme of HSPPC-96 vaccination for treating newly diagnosed GBM. IFN- $\gamma$ release ELISPOT, IFN- $\gamma$ release enzyme-linked immunospot assay; TMZ, temozolomide; p.o., oral administration; s.c., subcutaneously; CTX, cyclophosphamide.

(23), and GBM $(12,13)$. Two previous studies from the laboratory of Andrew T. Parsa $(12,13)$ have shown the safety and efficacy of HSPPC-96 vaccination in treating recurrent GBM. However, few studies are yet available to evaluate HSPPC-96 vaccination as a first-line therapy in the treatment of newly diagnosed malignancies. Here, we investigated the safety and preliminary efficacy of HSPPC-96 vaccination in conjunction with other first-line tumor therapies (e.g., Stupp's regimen, ref. 1) in newly diagnosed GBM.

Consistent with the results of HSPPC-96 vaccination in recurrent GBM $(12,13)$ and other malignancies (20-23), the vaccine was well-tolerated in newly diagnosed GBM patients. No grade 3 or 4 vaccine-related adverse events were reported during treatment (Table 2). Regarding whether HSPPC-96 vaccination will increase the toxicity of TMZ-based chemotherapy, we did not observe any increased toxicity compared with historical controls (Stupp's cohort, ref. 1). However, our study was a single-arm, uncontrolled trial. A definite answer to the question requires a large clinical trial with randomized controls in the future.

Stupp's regimen has been the standard postsurgical treatment for newly diagnosed GBM since it was introduced in 2005 (1). In Stupp's cohort, PFS was 53.9\% at 6 months, the median PFS time was 6.9 months, and the median OS time was 14.6 months (1). We extracted the GBM data sets in the The Cancer Genome Atlas (TCGA) database (http://cancergenome.nih.gov/). Of the 159 newly diagnosed GBM cases in TCGA with available postoperative therapy information, 66 patients (TCGA cohort) received the standard Stupp regimen. Median PFS and OS were 8.1 months and 16.0 months, respectively, in the TCGA cohort. Pei Yang et al. (24) have reported outcomes in a cohort of 274

Table 2. Adverse events in patients receiving the HSPPC-96 vaccine

\begin{tabular}{|c|c|c|c|}
\hline Adverse events & Grades 1-2 & Grades 3-5 & Attributable \\
\hline \multicolumn{4}{|l|}{ Constitutional } \\
\hline Fatigue & $4(20 \%)$ & 0 & Vaccine \\
\hline Anorexia & $1(5 \%)$ & 0 & Unrelated \\
\hline Fever & $1(5 \%)$ & 0 & Vaccine \\
\hline \multicolumn{4}{|l|}{ Dermatological } \\
\hline Pruritus & $1(5 \%)$ & 0 & Vaccine \\
\hline Gastrointestinal & $5(25 \%)$ & 0 & Chemotherapy \\
\hline \multicolumn{4}{|l|}{ Hematological } \\
\hline Leukopenia & $10(50 \%)$ & 0 & Chemotherapy \\
\hline \multicolumn{4}{|l|}{ Neurological } \\
\hline Seizure & $1(5 \%)$ & 0 & Unrelated \\
\hline Focal deficit & $3(15 \%)$ & $1(5 \%)$ & Unrelated \\
\hline Mood & $1(5 \%)$ & 0 & Unrelated \\
\hline
\end{tabular}
GBM patients from our center. In Yang's cohort, PFS was $71 \%$ at 6 months and median PFS and OS were 10.7 and 17.8 months, respectively, among 229 patients who received Stupp's regimen. Compared with these historical results, higher PFS at 6 months (89.5\%) and longer OS (31.4 months) were observed in our vaccinated GBM patients. Therefore, the HSPPC-96 vaccination would improve the outcomes for GBM patients who receive the standard Stupp regimen concurrently.

IDH mutations and meMGMTs are the two most predominant genetic characteristics that predict favorable outcomes in GBM patients (13-16). Therefore, the improved outcomes we found might have been biased by a high prevalence of these genetic features in our patients. However, only 2 of our patients exhibited IDH mutations or meMGMT (Table 1). The proportion of IDH mutations in our study (12.5\%) was not significantly different from the proportions in the TCGA cohort $(9.5 \%$; 
A Progression-free Survival

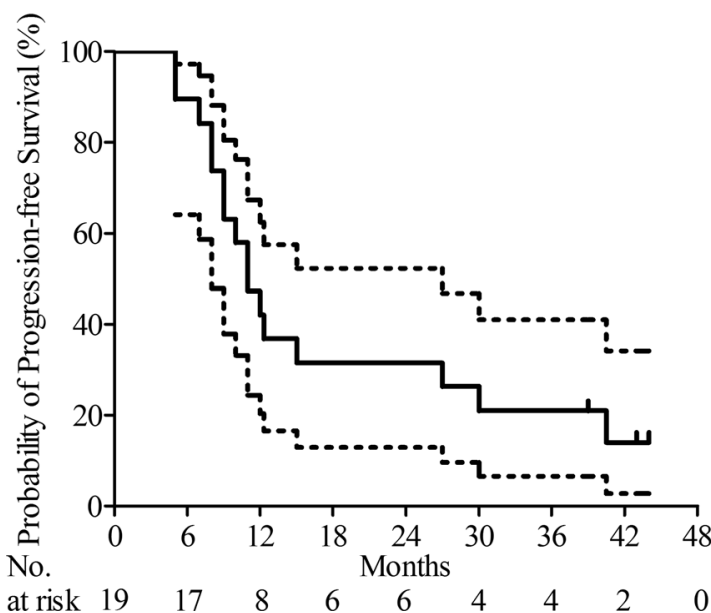

B Overall Survival

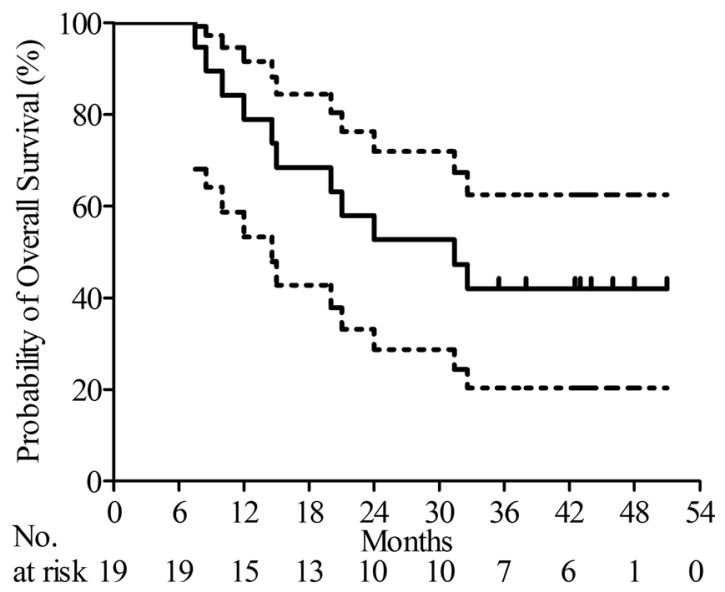

Figure 3. Clinical activity after HSPPC-96 vaccination. Kaplan-Meier estimates of (A) progression-free survival and (B) overall survival in 19 patients receiving the HSPPC-96 vaccine for the treatment of newly diagnosed GBMs. Vertical lines indicate time points at which patients were censored. Dotted lines indicate the $95 \% \mathrm{Cl}$.

Fisher's exact test, $P=0.661)$ or Yang's cohort (24) $(21.1 \%$; Fisher's exact test, $P=0.538)$. Significantly fewer of our patients $(12.5 \%)$ had a meMGMT than did patients in Stupp's cohort (1) (43.4\%; Fisher's exact test, $P=0.026$ ), the TCGA cohort (50\%; Fisher's exact test, $P=0.009$ ), and Yang's cohort (24) (39.9\%; Fisher's exact test, $P=0.033$ ). Therefore, the improved outcomes in this study were achieved in a GBM cohort harboring a low frequency of meMGMT. In addition, 13 patients did not have IDH mutations or meMGMT in this study. Median PFS was 10.0 months $(95 \%$ CI, 6.5-13.5) and OS was 21.0 months $(95 \%$ CI, 10.4-31.6) in these 13 patients. Median OS time was longer than that reported in Stupp's cohort (12.7 months in the un-meMGMT group) (14), the TCGA cohort (15.1 months in the wild-type IDH/un-meMGMT group), and Yang's cohort (15.0 months in the wild-type IDH/ un-meMGMT group).

Consistent with the improvements in patient outcomes, TSIR was increased after vaccination (Figure 4). Many studies have revealed the close association between TSIR and improved survival in cancer patients (25-27). Our study showed that TSIR after vaccination, instead of TSIR before vaccination, correlated with good survival in vaccinated patients (Figure 5, Table 3, and Supplemental Figure 1). Therefore, the favorable prognosis we observed in patients was very likely due to improved TSIR, which was stimulated by the HSPPC-96 vaccination.

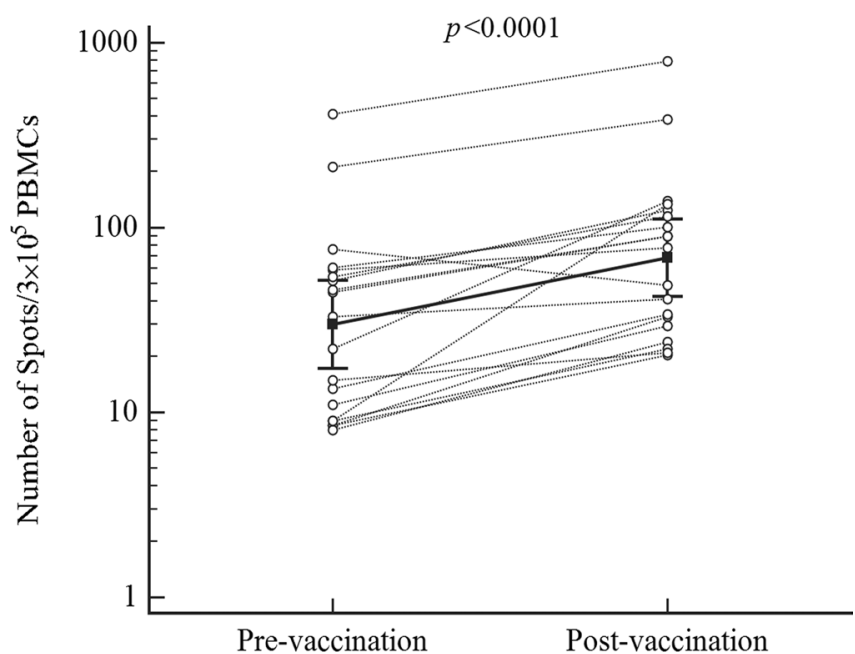

Pre-vaccination
Post-vaccination
Figure 4. Tumor-specific immune response before and after HSPPC-96 vaccination. Tumor-specific immune response was evaluated by the number of stimulated peripheral blood mononuclear cells (PBMCs) in response to autologous tumor lysate (measured by an IFN- $\gamma$ release enzyme-linked immunospot assay). Open circles represent the mean amounts from two repeated assays of each patient. Solid squares indicate the mean amounts of all included patients. Paired $t$ test was applied to evaluate the difference $(n=19)$. Error bars denote $95 \% \mathrm{Cl}$. 
A

- Pre-vaccination $\quad$ Post-vaccination

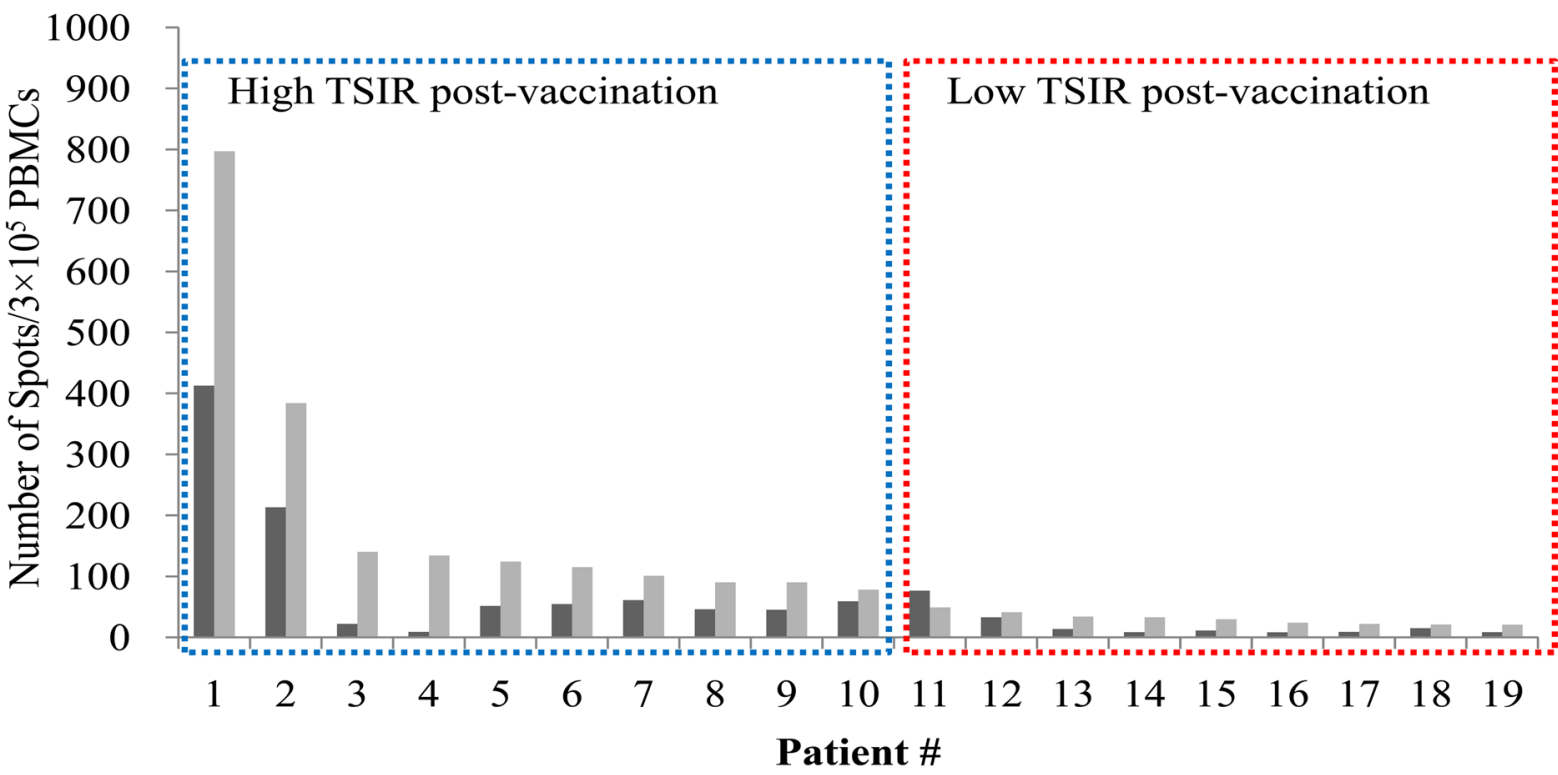

B Progresion-free Survival

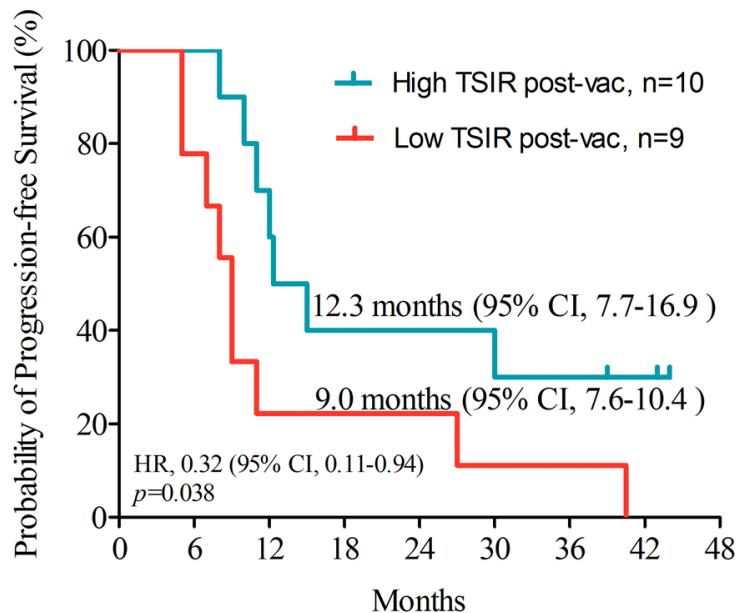

C Overall Survival

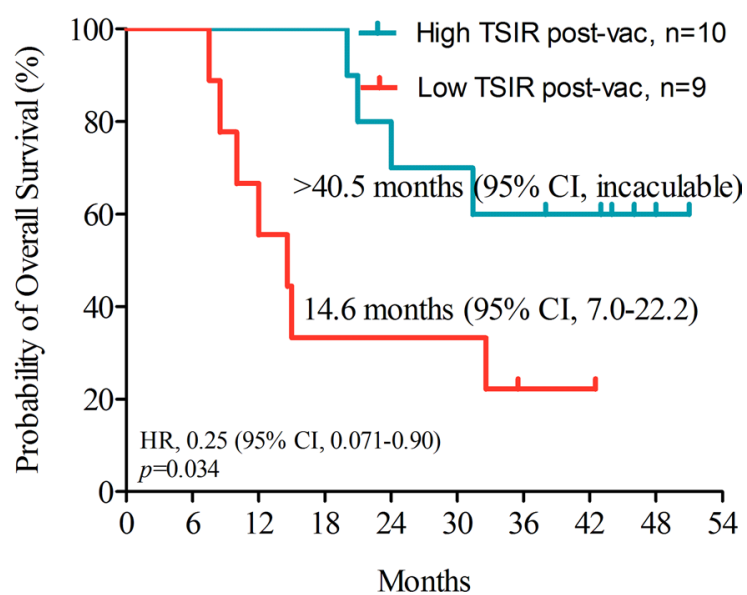

Figure 5. Association between clinical activity and immunological response. (A) Based on the tumor-specific immune response (TSIR) after vaccination, patients were divided into a high TSIR after vaccination (post-vac) group (TSIR post-vac $\geq$ median) and a low TSIR after vaccination group (TSIR post-vac < median). (B and C) Kaplan-Meier estimates of (B) progression-free survival and (C) overall survival in 19 GBM patients, divided into high and low TSIR post-vac groups. Log-rank test was applied to estimate the difference. Vertical lines indicate time points at which patients were censored.

However, because of the limited sample size and the phase I design of our study, our results are preliminary. Some baseline clinical characteristics, including a 100\% total resection rate, a good Karnofsky performance score, and a relatively young median age (Table 1), could provide a survival benefit and then bias the improved outcomes for this cohort of patients. Therefore, a large sample size cohort with a randomized controlled design is required to further determine the safety and efficacy of the vaccine for this deadly brain tumor. Meanwhile, TSIR after vaccination was a primary independent predictor for survival in the vaccinated patients. Further exploration is required to validate the role of TSIR after vaccination in deciding whether patients would benefit from the vaccine.

Conclusion. The use of HSPPC-96 vaccination in combination with the standard Stupp regimen is a safe and effective strategy with which to treat newly diagnosed GBM. Our study has laid down a solid foundation for further investigation of the safety and efficacy of this vaccine in a larger cohort of newly diagnosed GBM patients. 
Table 3. Cox regression model predicting median overall survival

\begin{tabular}{|c|c|c|c|}
\hline Parameter & Hazard ratio & $95 \% \mathrm{Cl}$ & $\boldsymbol{P}$ \\
\hline Sex, male vs. female & 5.56 & $0.72-42.74$ & 0.099 \\
\hline 70 & 0.52 & $0.08-3.57$ & 0.508 \\
\hline 80 & 0.10 & $0.01-1.11$ & 0.060 \\
\hline 90 & 1.00 & & \\
\hline
\end{tabular}

\section{Methods}

\section{Patients}

Eligible patients were at least 18 years and at most 75 years of age; had received a $\geq 80 \%$ microsurgical resection of a contrast-enhancing tumor; had a newly diagnosed supratentorial GBM confirmed with histology; had a Karnofsky performance status of $\geq 70 \%$ before vaccination; had the life expectancy to complete the standard Stupp regimen of radiotherapy plus concomitant and adjuvant TMZ (1); and had adequate organ function. Patients were excluded if they met any of the following criteria: serious unstable systemic diseases, known human immunodeficiency virus infection, active hepatitis viral infection, chronic diseases requiring steroids or immunosuppressive treatments, allergic constitution, or a tumor insufficient to create at least 6 doses of the vaccine.

\section{Study designs}

This study consisted of an open-label, single-arm, phase I clinical trial, which was performed at one center, the Beijing Tiantan Hospital. All selected patients underwent microsurgical resections and intraoperative collection of their tumor tissues for the production of vaccine (Figure 1). All patients underwent postoperative contrast-enhanced MRI of brain within 72 hours of surgery to evaluate the extent of resection. After completion of the standard course of radiotherapy plus concurrent TMZ chemotherapy, each patient had a repeat MRI and a clinical evaluation. If patients were eligible for inclusion, they were enrolled for vaccine administration (Figure 1). The scheme of HSPPC-96 vaccination is shown in Figure 2. The first vaccination was given concurrently with the beginning of adjunctive TMZ chemotherapy. Vaccines were administered via subcutaneous injection in $25-\mu \mathrm{g}$ doses every week for 6 weeks. Cyclophosphamide (400 $\mathrm{mg}$ ) was given through intravenous injection before each vaccine injection. Surveillance MRIs and clinical evaluations were performed to screen for progression every 3 months in the first 6 months after resection and every 6 months thereafter. Progression was defined according to the RANO criteria (28). Salvage therapy after progression was given at the discretion of the patient's neuro-oncologist. Patients were followed from surgery to progression or death to determine the duration of PFS or OS.

The primary endpoint was PFS at 6 months and the frequency of adverse events during the course of the vaccinations, occurring from the inclusion of the patient in the trial until 1 month after the last TMZ chemotherapy. Secondary endpoints included OS and PFS at the end of the study and immunological response during the course of the vaccinations. Adverse events were evaluated using the Common Terminology Criteria for Adverse Events (version 3.0, the National Cancer Institute, USA).

The genetic mutations of IDH and the methylation status of the MGMT promoter were also determined in the residual tumor samples of 16 patients. Mutations were sequenced and analyzed by Beijing Genetron Health Co. Ltd.

\section{HSPPC-96 vaccine generation}

After histological confirmation of GBM, tumor tissue was fresh frozen and shipped to Cure \& Sure Biotech Co. Ltd. for vaccine generation. The vaccine generation procedure has been described previously (29). A minimum of $4 \mathrm{~g}$ tumor tissue was required to generate six $25-\mu \mathrm{g}$ vaccine doses. 
Vaccine production followed good manufacturing practice guidelines. Vaccine quality was assessed by postproduction tests, including measurement of vaccine purity, vaccine concentration, endotoxin, and microbial content.

\section{Immunological response monitoring}

PBMC samples for immunological monitoring. A total of $10 \mathrm{ml}$ peripheral blood was taken from each included patient before the first injection (baseline, before vaccination) and after the fifth injection (after vaccination) (Figure 2). PBMCs were extracted from whole blood by Ficoll-Paque Plus (GE Healthcare Life Sciences) gradient centrifugation.

Autologous tumor lysate. GBM tumor tissue $(1 \mathrm{~g})$ from each patient was homogenized in $8 \mathrm{ml}$ of $30 \mathrm{mmol}$ $\mathrm{NaHCO}_{3}$ by mechanical processing. After centrifugation, the resultant supernatant was obtained as tumor lysate.

ELISPOT analysis. TSIR was evaluated by an IFN- $\gamma$ release ELISPOT assay using previously described PBMCs (Figure 2). T cell reactivity in PBMCs was evaluated in response to autologous tumor lysate. IFN- $\gamma$ release ELISPOT assay was performed at Cure \& Sure Biotech Co. Ltd. Each assay was repeated once.

The IFN- $\gamma$ release ELISPOT assay was described previously (30). Briefly, sterile 96-well polyvinylidene fluoride plates (MilliporeSigma) were coated with $100 \mu 1 /$ well IFN- $\gamma$ monoclonal antibody at $15 \mu \mathrm{g} / \mathrm{ml}$ (clone 1-D1K, Mabtech) and incubated for 2 hours at $37^{\circ} \mathrm{C}$. Plates were washed 5 times with PBS and then blocked with $200 \mu 1 /$ well complete media ( $10 \%$ fetal calf serum) for 1 hour at $37^{\circ} \mathrm{C}$. After removal of the complete media, autologous tumor lysate was added to a well at a concentration of 20 $\mu \mathrm{g} / \mathrm{ml}$. PMA (MilliporeSigma) was used as a positive control at a final concentration of $10 \mu \mathrm{g} / \mathrm{ml}$. The well without added tumor lysate or PMA was used as a negative control well. IL-2 (MilliporeSigma) was also added to each well at a final concentration of $50 \mathrm{IU} / \mathrm{ml}$. A total of $3 \times 10^{5} \mathrm{PBMCs}$ was added to each well and incubated for $16-24$ hours at $37^{\circ} \mathrm{C}$ in $5 \% \mathrm{CO}_{2}$. After being washed with PBS 5 times, the wells were incubated in $100 \mu \mathrm{l} /$ well of $1 \mu \mathrm{g} / \mathrm{ml}$ biotinylated IFN- $\gamma$ monoclonal antibody (clone 7-B6-1-Biotin, Mabtech) for 2 hours at room temperature. After washing 5 times, $100 \mu 1 /$ well streptavidin alkaline phosphatase (Streptavidin-ALP, Mabtech), diluted 1:1,000 in PBS/0.5\% FCS, was added to the wells. After a 1-hour incubation at room temperature, the plates were washed and developed with $100 \mu \mathrm{l} /$ well substrate (BCIP-NBT, Bio-Rad). Development was continued until dark spots appeared (up to 30 minutes). Spots were counted by a computer-assisted ELISPOT reader (Cellular Technology Ltd.). To calculate the number of PBMCs responding to tumor lysate by IFN- $\gamma$ release, the background (the number of IFN- $\gamma$ spots in the negative control well) was subtracted.

\section{Statistics}

The safety population was 20 patients, all of whom received at least 1 dose of the HSPPC-96 vaccine and were included in the description of baseline characteristics and safety analyses (Tables 1 and 2). The efficacy population was 19 patients, all of whom had a complete follow-up after 6 doses of the vaccine, and they were included in the evaluation of immunological response and clinical activity. Categorical data were compared using a $\chi^{2}$ test or Fisher's exact test. Continuous data were compared using the 2-tailed Student's $t$ test. The Kaplan-Meier analysis was used to estimate the OS and PFS time of included patients. The log-rank test was applied to estimate difference in OS and PFS time between groups. A Cox regression model was fitted to select the independent prognostic factors. A 2-tailed $P$ value of less than 0.05 was considered significant. All statistics were analyzed using SPSS version 20 (IBM).

\section{Study approval}

All patients provided written informed consent before participation in the trial. The protocol was approved by the ethics committee at Beijing Tiantan Hospital (JS2012-001-03) and was registered at ClinicalTrials.gov (NCT02122822) and http://www.chictr.org.cn/enindex.aspx (ChiCTR-ONC-13003309).

\section{Author contributions}

ZG conceived, designed, and supervised the study; NJ, YZ, YL, JX, YW, SH, and ZG acquired data; and NJ, YZ, and ZG analyzed and interpreted data and wrote, reviewed, and/or revised the manuscript. 


\section{Acknowledgments}

This work was supported by grants from the National Key Technology Research and Development Program of the Ministry of Science and Technology of China (2014BAI04B01, 2014BAI04B02), the Beijing Natural Science Foundation (7164253), the Beijing Talents Fund (2014000021469G257), and the Shenzhen Science and Technology Innovation Committee (JSGG20170413151359491).

Address correspondence to: Zhixian Gao, Department of Neurosurgery, Beijing Tiantan Hospital, Capital Medical University, 6 Tian Tan Xi Li, Dong cheng district, Beijing, China, 100050. Phone: 86.10.6709.8402; Email: btth_ns@163.com.

1. Stupp R, et al. Radiotherapy plus concomitant and adjuvant temozolomide for glioblastoma. N Engl J Med. 2005;352(10):987-996.

2. Stupp R, et al. Effects of radiotherapy with concomitant and adjuvant temozolomide versus radiotherapy alone on survival in glioblastoma in a randomised phase III study: 5-year analysis of the EORTC-NCIC trial. Lancet Oncol. 2009;10(5):459-466.

3. Hoos A. Development of immuno-oncology drugs - from CTLA4 to PD1 to the next generations. Nat Rev Drug Discov. 2016;15(4):235-247.

4. D'Errico G, Machado HL, Sainz B. A current perspective on cancer immune therapy: step-by-step approach to constructing the magic bullet. Clin Transl Med. 2017;6(1):3.

5. Sampson JH, Maus MV, June CH. Immunotherapy for brain tumors. J Clin Oncol. 2017;35(21):2450-2456.

6. Tivnan A, Heilinger T, Lavelle EC, Prehn JH. Advances in immunotherapy for the treatment of glioblastoma. J Neurooncol. 2017;131(1):1-9.

7. Reardon DA, et al. Immunotherapy advances for glioblastoma. Neuro-oncology. 2014;16(11):1441-1458.

8. Suto R, Srivastava PK. A mechanism for the specific immunogenicity of heat shock protein-chaperoned peptides. Science. 1995;269(5230):1585-1588.

9. Tamura Y, Peng P, Liu K, Daou M, Srivastava PK. Immunotherapy of tumors with autologous tumor-derived heat shock protein preparations. Science. 1997;278(5335):117-120.

10. Zheng H, Dai J, Stoilova D, Li Z. Cell surface targeting of heat shock protein gp96 induces dendritic cell maturation and antitumor immunity. J Immunol. 2001;167(12):6731-6735.

11. Weller M, et al. Vaccine-based immunotherapeutic approaches to gliomas and beyond. Nat Rev Neurol. 2017;13(6):363-374.

12. Crane CA, et al. Individual patient-specific immunity against high-grade glioma after vaccination with autologous tumor derived peptides bound to the 96 KD chaperone protein. Clin Cancer Res. 2013;19(1):205-214.

13. Bloch O, et al. Heat-shock protein peptide complex-96 vaccination for recurrent glioblastoma: a phase II, single-arm trial. Neuro-oncology. 2014;16(2):274-279.

14. Hegi ME, et al. MGMT gene silencing and benefit from temozolomide in glioblastoma. N Engl J Med. 2005;352(10):997-1003

15. Wick W, et al. MGMT testing--the challenges for biomarker-based glioma treatment. Nat Rev Neurol. 2014;10(7):372-385.

16. Waitkus MS, Diplas BH, Yan H. Isocitrate dehydrogenase mutations in gliomas. Neuro-oncology. 2016;18(1):16-26.

17. Nduom EK, Weller M, Heimberger AB. Immunosuppressive mechanisms in glioblastoma. Neuro-oncology. 2015;17 Suppl 7:vii9-vii14

18. Mangani D, Weller M, Roth P. The network of immunosuppressive pathways in glioblastoma. Biochem Pharmacol. 2017;130:1-9.

19. Filley AC, Dey M. Dendritic cell based vaccination strategy: an evolving paradigm. J Neurooncol. 2017;133(2):223-235.

20. Testori A, et al. Phase III comparison of vitespen, an autologous tumor-derived heat shock protein gp 96 peptide complex vaccine, with physician's choice of treatment for stage IV melanoma: the C-100-21 Study Group. J Clin Oncol. 2008;26(6):955-962.

21. Eton O, et al. Autologous tumor-derived heat-shock protein peptide complex-96 (HSPPC-96) in patients with metastatic melanoma. J Transl Med. 2010;8:9.

22. Jonasch $\mathrm{E}$, et al. Vaccination of metastatic renal cell carcinoma patients with autologous tumour-derived vitespen vaccine: clinical findings. Br J Cancer. 2008;98(8):1336-1341.

23. Mazzaferro V, et al. Vaccination with autologous tumor-derived heat-shock protein gp 96 after liver resection for metastatic colorectal cancer. Clin Cancer Res. 2003;9(9):3235-3245.

24. Yang P, et al. IDH mutation and MGMT promoter methylation in glioblastoma: results of a prospective registry. Oncotarget. 2015;6(38):40896-40906.

25. Liu FF, et al. The specific immune response to tumor antigen CP1 and its correlation with improved survival in colon cancer patients. Gastroenterology. 2008;134(4):998-1006.

26. Schirrmacher V, Fournier P, Schlag P. Autologous tumor cell vaccines for post-operative active-specific immunotherapy of colorectal carcinoma: long-term patient survival and mechanism of function. Expert Rev Vaccines. 2014;13(1):117-130.

27. GuhaThakurta D, et al. Humoral immune response against nontargeted tumor antigens after treatment with sipuleucel-t and its association with improved clinical outcome. Clin Cancer Res. 2015;21(16):3619-3630.

28. Wen PY, et al. Updated response assessment criteria for high-grade gliomas: response assessment in neuro-oncology working group. J Clin Oncol. 2010;28(11):1963-1972.

29. Meng SD, Song J, Rao Z, Tien P, Gao GF. Three-step purification of gp96 from human liver tumor tissues suitable for isolation of gp96-bound peptides. J Immunol Methods. 2002;264(1-2):29-35.

30. Yan X, et al. Regulatory T-cell depletion synergizes with gp96-mediated cellular responses and antitumor activity. Cancer Immunol Immunother. 2011;60(12):1763-1774. 